This document is the accepted manuscript version of the following article:

Dascalu, M., Dünki, S. J., Quinsaat, J. -E. Q., Ko, Y. S., \& Opris, D. M. (2015). Synthesis of silicone elastomers containing trifluoropropyl groups and their use in dielectric elastomer transducers. RSC Advances, 5(126), 104516-104523.

http://doi.org/10.1039/C5RA16132K

\title{
ARTICLE
}

\section{Synthesis of silicone elastomers containing trifluoropropyl groups and their use in dielectric elastomer transducers}

\begin{abstract}
Mihaela Dascalu, ${ }^{a}+$ Simon J. Dünki, ${ }^{a, b}$ Jose-Enrico Q. Quinsaat, ${ }^{a, b}$ Yee Song Ko, ${ }^{a, b}$ Dorina M. Opris ${ }^{a *}$
Vinyl end-functionalized polysiloxanes $\mathbf{P}_{\mathbf{x}}$ containing varying mol\% of trifluoropropyl groups $(\mathbf{x})$ were prepared starting from 1,3,5-tris(3,3,3-trifluoropropyl)-1,3,5-trimethylcyclotrisiloxane $\left(F_{3}\right)$ and octamethylcyclotetrasiloxane $\left(D_{4}\right)$ via anionic polymerization in the presence of tetramethylammonium hydroxide (TMAH) and 1,3-divinyl-1,1,3,3-tetramethyldisiloxane endcapping reagent. Their structures were determined by ${ }^{1} \mathrm{H}$ NMR spectroscopy and their molecular weights and distributions were measured by GPC. The various $\mathbf{P}_{\mathbf{x}}$ were cross-linked in thin films via hydrosilylation of the vinyl groups with tetrakis(dimethylsiloxy)silane cross-linker in the presence of Karstedt catalyst. The mechanical, dielectric and electromechanical properties of the prepared films were investigated. An increase in the permittivity $\left(\varepsilon^{\prime}\right)$ with increasing content of polar trifluoropropyl groups was observed with a maximum value of $\varepsilon^{\prime}=6.4$ for $\mathbf{P}_{58}(\mathbf{0})$. A maximum lateral actuation strain of $5.4 \%$ at an electric field as low as $7.8 \mathrm{~V} / \mu \mathrm{m}$ was measured for a material prepared by cross-linking $\mathbf{P}_{53}$.
\end{abstract}

\section{Introduction}

Cross-linked polydimethylsiloxanes (silicones) are presently intensively explored as dielectrics in dielectric elastomer transducers (DET) ${ }^{1,2}$ DET are stretchable capacitors that elongate when charged and can act as actuators, generators, and sensors. ${ }^{3}$ A large variety of applications were proposed, which range from valves, pumps, wave generators, pressure sensors, to muscle replacements to name a few. ${ }^{4}$

Any elastomers can in principle be used as dielectric in DET, however silicones are most often used mainly due to their fast response time, low viscoelastic loss, and low $T_{g}$ which allow operation at different frequencies and temperatures. Silicones have a high dielectric strength, high gas permeability and hydrophobicity which afford them with humidity tolerance and a low water uptake. ${ }^{5}$ Silicone actuators can have large actuation strains and high energy densities, but rather high electric fields have to be used. ${ }^{6,7}$ More efficient silicones that actuate at low electric fields might pave the way for the application of this technology in the area of medicine, e.g. as implantable devices, where the maximum allowed operation voltage

\footnotetext{
a. Empa, Swiss Federal Laboratories for Materials Science and Technology, Laboratory for Functional Polymers, Ueberlandstr. 129, CH-8600, Dübendorf, Switzerland, E-mail: dorina.opris@empa.ch.

${ }^{b .}$ Ecole Polytechnique Fédérale de Lausanne (EPFL), Institut des matériaux, Station 12, CH 1015, Lausanne, Switzerland.

+ Present address: Petru Poni Institute of Macromolecular Chemistry, Aleea Grigore Ghica Voda 41A, Iasi, 700487, Romania

Electronic Supplementary Information (ESI) available: ${ }^{1} \mathrm{H}$ NMR and IR spectra, GPC data, DSC curves, self-healing of an actuator. See DOI: 10.1039/x0xx00000x
}

is below $24 \mathrm{~V}^{8}$ The actuation strain can be increased when the thickness of the dielectric film is reduced and when soft high permittivity elastomers are used as dielectric. Another possibility to enhance the actuation strain is to use appropriately designed heterogeneous materials consisting of alternating soft isotropic and anisotropic layers. For such materials theoretical calculations predict a ten-fold enhancement of the electromechanical coupling. ${ }^{9}$

The $\varepsilon^{\prime}$ of polymers can be increased when blended with high permittivity ceramic ${ }^{10,11}$ or conductive particles. ${ }^{12-17}$ As fillers, polyaniline, ${ }^{12}$ encapsulated polyaniline in polydivinylbenzene, ${ }_{1}^{13}$ silver nanoparticles coated with a thin silica shell, ${ }^{14}$ carbon black, ${ }^{15}$ carbon nanotubes, ${ }^{16}$ and polythiophene ${ }^{17}$ have been used. An increase in the permittivity was observed with increasing volume fraction of conductive filler, but other properties like elasticity, elastic moduli, strain at break, dielectric loss, and dielectric breakdown were negatively affected. Furthermore, the migration of the conductive filler in an electric field and thus the long term stability of such composites are of concern especially when thermoplastic matrices are used. Ceramic fillers significantly increase the permittivity, but concentrations above 50 vol\% have to be used. The resulting materials tend to have poor mechanical properties and increased elastic moduli. The present work is focused on increasing the electromechanical response in an actuator by using soft high permittivity silicones.

Silicones have a rather low $\varepsilon^{\prime}$ of less than 3 and therefore high electric fields are required to induce actuation. It has been suggested that the $\varepsilon^{\prime}$ of polymers can be increased when modified with polar groups. ${ }^{18}$ 
When modified with nitroaniline and nitrobenzene moieties, silicones show $\varepsilon^{\prime}=6$ and $\varepsilon^{\prime}=8.5$ at high frequencies, respectively, ${ }^{19,20}$ while $\varepsilon^{\prime}$ $=4.7$ can be achieved when modified with chloropropyl groups. ${ }^{21} \mathrm{By}$ modifying the silicones with cyanopropyl groups at every repeat unit, polymers with $\varepsilon^{\prime}$ of about 18 were prepared. ${ }^{22,23}$ Recently, the synthesis of a silicone elastomer modified with polar nitrile groups was reported which combines $\varepsilon^{\prime}$ of about 10 , low elastic moduli and a strain at break of $260 \% .{ }^{24}$ Commercial fluorinated silicones also show a high permittivity and were used by Perline et al. as dielectric in actuators. ${ }^{25}$ Unfortunately, the reported materials are commercial products and therefore no data about their chemical composition is available. More recently, Böse et al. used a trifluoropropyl $\left(\mathrm{CF}_{3}\right)$ modified silicone oil as softening agent in a polydimethylsiloxane matrix and obtained a material with $\varepsilon^{\prime}=5.5$, a strain at break as high as $400 \%$, and a maximum actuation strain of $7.5 \%$ at $14 \mathrm{~V} / \mu \mathrm{m} .{ }^{26}$ The polar $\mathrm{CF}_{3}$ modified silicone oil was mixed together with a nonpolar polydimethylsiloxane and thus the resulting materials might undergo phase separation. Furthermore, the amount of $\mathrm{CF}_{3}$ modified silicone used in the silicone matrix was limited to $45 \%$ which represents about 28 mol\% of $\mathrm{CF}_{3}$ groups in the material. A farther increase of the $\mathrm{CF}_{3}$ content resulted in very soft materials which were difficult to process in thin films.

The aim of this work was to synthesize high molecular weight polysiloxanes $\mathbf{P}_{\mathbf{x}}$ containing different mol\% of $\mathrm{CF}_{3}$ groups as well as reactive end-groups which allow them to be cross-linked in thin films. Homogenous materials were prepared, for which the dielectric, mechanical, and electromechanical properties were investigated as function of $\mathrm{CF}_{3}$ content.

\section{Experimental Section}

\section{Materials and Characterization}

1,3,5-Tris(trifluoropropylmethyl) cyclotrisiloxane $\left(F_{3}\right)$ was purchased from EuroLab Limited. Octamethylcyclotetrasiloxane $\left(D_{4}\right)$ and tetrakis(dimethylsiloxy)silane were purchased from $A B C R$, 1,3-divinyl1,1,3,3-tetramethyldisiloxane (EB), TMAH (25 wt\% solution in methanol) and platinum(0)-1,3-divinyl-1,1,3,3-tetramethyldisiloxane complex solution in xylene ( $\mathrm{Pt} \sim 2 \mathrm{wt}$. \%) were purchased from SigmaAldrich. Prior to use $D_{4}$ and $F_{3}$ were dried over calcium hydride, and TMAH was dried by azeotropic distillation. All other chemicals were used as received. Acrylic foil VHB from $3 \mathrm{M}$ was used.

FTIR spectra were recorded on a Bruker Vertex 70 ATR FT-IR spectrometer at room temperature. ${ }^{1} \mathrm{H}$ NMR spectra were recorded on a Bruker Avance III 400 NMR spectrometer using a $5 \mathrm{~mm}$ BBO Prodigy $^{\mathrm{TM}}$ CryoProbe at $400.18 \mathrm{~Hz}$. GPC was conducted using an Agilent 1100 Series HPLC (Columns: serial coupled PSS SDV $5 \mathrm{u}$, 100A and PSS SDV $5 \mathrm{u}, 1000 \mathrm{~A}$, Detector: DAD, $235 \mathrm{~nm}$ and $360 \mathrm{~nm}$; refractive index) with THF solvent using polydimethylsiloxane and toluene as internal standards. Thermogravimetric analysis (TGA) was conducted on a Perkin Elmer TGA7 at a heating rate of $20{ }^{\circ} \mathrm{C} \mathrm{min}{ }^{-1}$ under a nitrogen gas flow up to $800{ }^{\circ} \mathrm{C}$. Differential scanning calorimetry (DSC) measurements were conducted on a Perkin Elmer DSC 8000. The mechanical properties were investigated with a Zwick Z010 tensile test machine with a crosshead speed of $200 \mathrm{~mm} \mathrm{~min}^{-1}$ (strain rate of $1111.1 \% / \mathrm{min}$ ). Tensile test specimens with a gauge width of $2 \mathrm{~mm}$ and a gauge length of $18 \mathrm{~mm}$ were prepared by die cutting. The strain was determined using a traverse moving sensor. The curves were averaged from 2-4 different specimens (See ESI). The tensile modulus was determined from the slope of the stressstrain curves using a linear fit to the data points within $10 \%$ strain. The strain at break was calculated by taking the average of the different measurements. DMA spectra were measured on a ARES Rheometer from TA instruments with a parallel plate setup $(25 \mathrm{~mm}$ plate diameter), a strain of $5 \%$ and $1 \mathrm{~N}$ preforce. Dielectric measurements were conducted on a Novocontrol Alpha-A Frequency Analyzer using a Hewlett Packard 16451B Dielectric test fixture as electrodes. Shielded electrodes with a diameter of $5 \mathrm{~mm}$ were used and the sample thickness was determined by using the built in micrometer screw. The probing voltage was 1RMSV (root mean square voltage). Actuator tests were performed using circular membrane actuators, for which the films were fixed between two circular frames of $25 \mathrm{~mm}$. Circular electrodes ( $8 \mathrm{~mm}$ diameter) of carbon black powder were applied to each side of the film. A high voltage amplifier Trek Model 5/80 (up to $5600 \mathrm{~V}$ ) was used for actuator tests. The voltage was increased by $100 \mathrm{~V}$ every $2 \mathrm{~s}$. The actuation strain was measured optically as the extension of the diameter of the electrode area via a digital camera, using an edge detection tool of a LabView program to detect the boundary between the black electrode area and brighter film.

\section{General procedure for the synthesis of $P_{x}$}

A solution of TMAH in methanol $(73 \mu \mathrm{L})$ was added to a three-neck 50 $\mathrm{mL}$ round-bottom flask and dried by azeotropic distillation. $\mathrm{D}_{4}, \mathrm{~F}_{3}$ and EB were added simultaneously to the dried TMAH. For the amounts of reagents used, see Table 1 . The reaction mixture was heated to $100 \pm 2{ }^{\circ} \mathrm{C}$ under nitrogen for $4.5 \mathrm{~h}$. Then the reaction mixture was heated to $160{ }^{\circ} \mathrm{C}$ and kept at this temperature for $2 \mathrm{~h}$ to deactivate the initiator. Finally, the unreacted cycles were distilled at $180-200{ }^{\circ} \mathrm{C}$ under vacuum. The residue was washed with ethanol and then dried in an oven at $100{ }^{\circ} \mathrm{C}$ to obtain $\mathbf{P}_{\mathbf{x}} \cdot{ }^{1} \mathrm{H}$ NMR $\left(400 \mathrm{MHz}, \mathrm{CDCl}_{3}, \delta\right): 6.20$ 5.71 (vinyl-H), $2.08\left(\alpha-\mathrm{CH}_{2}-\mathrm{CH}_{2}-\mathrm{CF}_{3}\right), 0.76\left(\beta-\mathrm{CH}_{2}-\mathrm{CH}_{2}-\mathrm{CF}_{3}\right), 0.1$ (Si$\mathrm{CH}_{3}$ ). The chemical composition of $\mathbf{P}_{\mathbf{x}}$ as well as its molar mass and distribution are given in Table 2.

Table 1 The amount of reagents used for the synthesis of $\mathbf{P}_{\mathbf{x}}$.

\begin{tabular}{cccccc}
\hline Entry & $\begin{array}{c}\mathrm{F}_{3} \\
\mathrm{~g}(\mathrm{mmol})\end{array}$ & $\begin{array}{c}\mathrm{D}_{4} \\
\mathrm{~g}(\mathrm{mmol})\end{array}$ & $\begin{array}{l}\mathrm{F}_{3} / \mathrm{D}_{4} \\
\mathrm{~mol} \\
\text { ratio }\end{array}$ & $\begin{array}{c}\mathrm{EB} \\
\mu \mathrm{L}(\mathrm{mmol})\end{array}$ & $\begin{array}{c}{ }^{\mathrm{a}} \mathrm{TMAH} \\
{[\mu \mathrm{L}]}\end{array}$ \\
\hline $\mathbf{P}_{\mathbf{2 8}}$ & $11.2(23.9)$ & $7.1(23.9)$ & 1 & $23(0.1)$ & 73 \\
$\mathbf{P}_{42}$ & $22.3(47.6)$ & $14.2(47.9)$ & 1 & $45(0.2)$ & 128 \\
$\mathbf{P}_{47}$ & $21.7(46.3)$ & $10.6(35.7)$ & 1.3 & $45(0.2)$ & 128 \\
$\mathbf{P}_{53}$ & $21.7(46.3)$ & $9.2(31.0)$ & 1.5 & $45(0.2)$ & 123 \\
$\mathbf{P}_{58}$ & $21.7(46.3)$ & $5.9(19.9)$ & 2.3 & $45(0.2)$ & 110 \\
\hline
\end{tabular}

${ }^{\mathrm{a}}$ A solution of $25 \%$ in methanol was used.

Table 2 Composition and $M_{n}$ of copolymers $\mathbf{P}_{\mathbf{x}}$ calculated from ${ }^{1} \mathrm{H}$ NMR, as well as the $M_{n}, M_{w}$, and PDI obtained from the GPC measurements.

\begin{tabular}{cccccccc}
\hline \multirow{3}{*}{ Entry } & \multicolumn{3}{c}{ NMR } & & \multicolumn{3}{c}{ GPC } \\
\cline { 2 - 3 } \cline { 6 - 8 } & ${ }^{\mathrm{a}} \mathrm{F}$ & ${ }^{\mathrm{b}} \mathrm{D}$ & $M_{\mathrm{n}}$ & & $M_{n}$ & $M_{w}$ & \\
{$[\mathrm{~mol} \%]$} & {$[\mathrm{mol} \%]$} & {$[\mathrm{g} / \mathrm{mol}]$} & & & {$[\mathrm{g} / \mathrm{mol}]$} & {$[\mathrm{g} / \mathrm{mol}]$} & $\mathrm{PDI}$ \\
\hline
\end{tabular}




\begin{tabular}{lllllll}
\hline $\mathbf{P}_{\mathbf{2 8}}$ & 28.3 & 71.7 & 61600 & 46800 & 101700 & 2.2 \\
$\mathbf{P}_{\mathbf{4 2}}$ & 41.8 & 58.2 & 118500 & 59520 & 140200 & 2.4 \\
$\mathbf{P}_{\mathbf{4 7}}$ & 47.1 & 52.9 & 13400 & 11600 & 18200 & 1.6 \\
$\mathbf{P}_{53}$ & 52.9 & 47.1 & 48500 & 34700 & 75900 & 2.2 \\
$\mathbf{P}_{58}$ & 57.5 & 42.5 & 47000 & 16500 & 48900 & 3.0 \\
\hline
\end{tabular}

${ }^{\mathrm{a}} \mathrm{F}$ represents trifluoropropyl and ${ }^{\mathrm{b}}$ represents dimethylsilyl.

\section{General Synthesis of Polysiloxane Elastomers Containing} Trifluoropropyl groups

To a homogenous mixture of $\mathbf{P}_{\mathbf{x}}$ and tetrakis(dimethylsiloxy)silane cross-linker (CL, 10 wt\% in toluene), a solution of Karstedt catalyst (1 vol\% in toluene) was added. This mixture was stirred for another 3 min and sonicated for $1 \mathrm{~min}$ to remove the air bubbles. To reinforce the elastomers, a dispersion of hexamethyldisilasane (HMDS) treated silica particles in toluene was used. Thin films were prepared by the doctor blade technique. The samples were aged at $80^{\circ} \mathrm{C}$ in oven for $24 \mathrm{~h}$. The resulting elastomers were named as: $\mathbf{P}_{\mathbf{x}}(\mathbf{y})$, where $\mathbf{P}_{\mathbf{x}}$ represents the starting copolymer and y represents the wt\% of silica used. Table 3 summarises the amounts of reagents used for the synthesis of $\mathbf{P}_{\mathbf{x}}(\mathbf{y})$.

Table 3 Amount of reagents used for the synthesis of $\mathbf{P}_{\mathbf{x}}(\mathbf{y})$.

\begin{tabular}{|c|c|c|c|c|c|}
\hline Sample & $\begin{array}{l}P_{x} \\
{[g]}\end{array}$ & $\begin{array}{l}{ }^{\mathrm{a}} \mathrm{CL} \\
{[\mu \mathrm{L}]}\end{array}$ & $\begin{array}{l}\text { molar ratio } \\
{[\text { vinyl /Si-H] }}\end{array}$ & $\begin{array}{c}{ }^{\mathrm{b}} \text { Karstedt cat. } \\
{[\mu \mathrm{L}]}\end{array}$ & $\begin{array}{l}{ }^{\mathrm{d}} \mathrm{SiO}_{2} \\
{[\mathrm{mg}]}\end{array}$ \\
\hline$P_{28}(0)$ & 0.5 & 53 & 1.1 & 32.4 & - \\
\hline$P_{28}(5)$ & 0.5 & 53 & 1.1 & 32.4 & 25 \\
\hline$P_{28}(10)$ & 0.5 & 53 & 1.1 & 32.4 & 50 \\
\hline $\mathbf{P}_{42}(0)$ & 2.0 & 111 & 1.1 & 67.5 & - \\
\hline $\mathbf{P}_{42}(\mathbf{5})$ & 2.0 & 111 & 1.1 & 67.5 & 100 \\
\hline$P_{42}(10)$ & 2.0 & 111 & 1.1 & 67.5 & 200 \\
\hline$P_{47}(0)$ & 0.5 & 62 & 4.5 & ' 18.7 & - \\
\hline$P_{47}(5)$ & 0.5 & 62 & 4.5 & ' 18.7 & 25 \\
\hline$P_{47}(10)$ & 0.5 & 62 & 4.5 & 'c18.7 & 50 \\
\hline$P_{53}(0)$ & 2.0 & 271 & 1.1 & 164.8 & - \\
\hline$P_{53}(5)$ & 2.0 & 271 & 1.1 & 164.8 & 100 \\
\hline$P_{57}(0)$ & 2.0 & 279 & 1.1 & 170.0 & - \\
\hline$P_{57}(5)$ & 2.0 & 279 & 1.1 & 170.0 & 100 \\
\hline
\end{tabular}

${ }^{\mathrm{a}} \mathrm{A} 10 \mathrm{wt} \%$ solution of tetrakis(dimethylsiloxy)silane in toluene was used; ${ }^{b} 1$ vol\% or ${ }^{c} 0.5$ vol\% of Karstedt catalyst in toluene was used; ${ }^{d}$ silica particles were dispersed in toluene prior use.

\section{Results and Discussions}

Synthesis and characterization of $\mathbf{P}_{\mathbf{x}}$

Vinyl end-functionalized polysiloxanes containing varying mol\% of $\mathrm{CF}_{3}$ groups $\mathbf{P}_{\mathbf{x}}$ were prepared starting from $\mathrm{F}_{3}$ and $\mathrm{D}_{4}$ via anionic bulk polymerization in the presence of TMAH catalyst and 1,3-divinyl1,1,3,3-tetramethyldisiloxane end-capping reagent (Scheme 1). TMAH was selected as initiator because it can be decomposed to inactive components at elevated temperatures, while the end capping reagent allowed us to functionalize $\mathbf{P}_{\mathbf{x}}$ with vinyl endgroups that should be further used for the cross-linking in thin films. The content of $\mathrm{CF}_{3}$ groups in $\mathbf{P}_{\mathbf{x}}$ was tuned by using different feed ratios of $D_{4}$ to $F_{3}$, while the molecular weight can be controlled by the amount of end-capping reagent used. $\mathbf{P}_{\mathbf{x}}$ were analysed by ${ }^{1} \mathrm{H}$ NMR, IR and GPC.

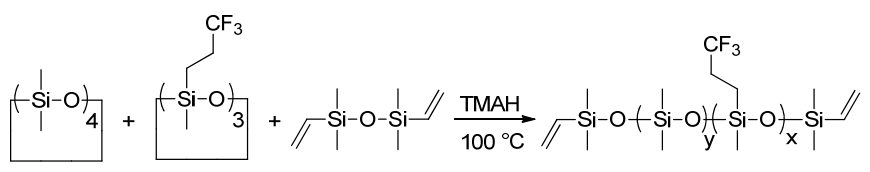

Scheme 1 Synthesis of vinyl end-functionalized polysiloxanes containing $\mathrm{CF}_{3}$ groups starting from $\mathrm{D}_{4}, \mathrm{~F}_{3}$, and 1,3-divinyl-1,1,3,3tetramethyldisiloxane end-blocker.

Fig. 1 shows the ${ }^{1} \mathrm{H}$ NMR spectra of $\mathbf{P}_{47}$. The signal at $\delta=0.10 \mathrm{ppm}$ is assigned to the methyl groups, the signals at $\delta=0.76 \mathrm{ppm}$ and $\delta=$ $2.08 \mathrm{ppm}$ correspond to the methylene units $\alpha$ - and $\beta$ - to $\mathrm{CF}_{3}$ groups. ${ }^{27}$ The signals between $\delta=5.71$ to $\delta=6.20 \mathrm{ppm}$ are attributed to the vinyl end-groups. ${ }^{28}$ The other copolymers $\mathbf{P}_{\mathbf{x}}$ show the same signals as those of $\mathbf{P}_{47}$, but the intensities of the signals are different (Figures S1-S5). The content of $\mathrm{CF}_{3}$ groups in $\mathbf{P}_{\mathbf{x}}$ was calculated using the integrals of the signal at $\delta=0.10 \mathrm{ppm}$ and that at $\delta=2.08$ of the methylene units $\alpha$ to $\mathrm{CF}_{3}$. Although $\mathrm{F}_{3}$ has a higher reactivity as compared to $\mathrm{D}_{4}$, the amount of $\mathrm{CF}_{3}$ units incorporated in the polymers was found to be lower than the feed ratios $F_{3}: D_{4}$. The reason for this might be the high temperature used for the decomposition of TMAH which might favour the depolymerization and formation of cyclic monomers containing $\mathrm{CF}_{3}$ units. Nevertheless, an increase in the content of $\mathrm{CF}_{3}$ units in $\mathbf{P}_{\mathbf{x}}$ with increasing the $F_{3}: D_{4}$ feed ratios was observed.

$M_{n}$ values were calculated by comparing the integral of the signal at $\delta=0.10 \mathrm{ppm}$ with the integral of the vinyl protons and found to be slightly higher than the $M_{n}$ values obtained from GPC (Figures S6-S10). ${ }^{29}$ Table 2 gives an overview of the chemical composition of $\mathbf{P}_{\mathbf{x}}$ as well as the molecular weight and its distribution as found by NMR (ESI Fig. S1-S5) and GPC measurements (see ESI).

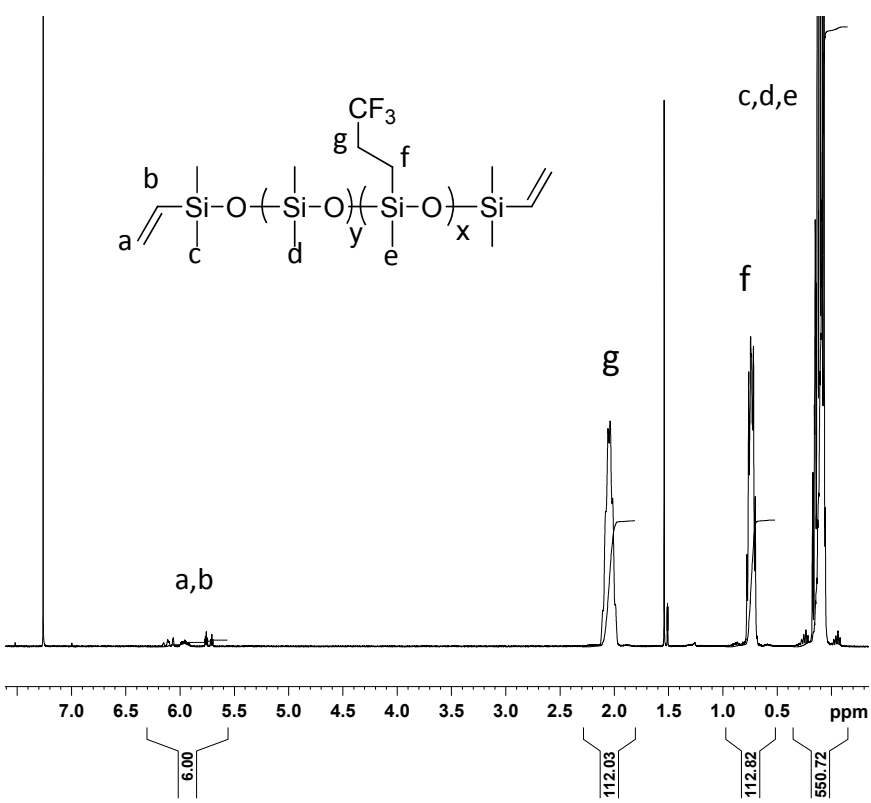

Fig. $1^{1} \mathrm{H}$ NMR spectrum of $\mathbf{P}_{47}$ in $\mathrm{CDCl}_{3}$ at room temperature. 
The infrared absorption spectra of $\mathbf{P}_{\mathrm{x}}$ are shown in Figure S11 (see ESI). The broad and strong absorption band from $1170 \mathrm{~cm}^{-1}$ to 1000 $\mathrm{cm}^{-1}$ in each spectrum is attributed to the $\mathrm{Si}-\mathrm{O}-\mathrm{Si}$ asymmetric stretching vibration. ${ }^{30,31}$ The absorption bands from $2964 \mathrm{~cm}^{-1}$ and $2909 \mathrm{~cm}^{-1}$ are assigned to the $\mathrm{C}-\mathrm{H}$ vibrations from $-\mathrm{CH}_{3}$ groups. The peak at $1264 \mathrm{~cm}^{-1}$ is assigned to the absorption due to vibration of $\mathrm{Si}-\mathrm{CH}_{3}$. Characteristic absorption peaks of $-\mathrm{CH}_{2} \mathrm{CH}_{2} \mathrm{CF}_{3}$ appeared at $1368 \mathrm{~cm}^{-1}$ (wagging $-\mathrm{CH}_{2}-$ ), $1315 \mathrm{~cm}^{-1}\left(\mathrm{CH}_{2}-\mathrm{CH}_{2}\right), 1208 \mathrm{~cm}^{-1}\left(-\mathrm{CF}_{3}\right)$, $1168 \mathrm{~cm}^{-1}$ ( $\mathrm{C}-\mathrm{H}$ bond in $-\mathrm{CH}_{2}-$ ) and $902 \mathrm{~cm}^{-1}\left(\mathrm{C}_{-}-\mathrm{CF}_{3}\right)$. An absorption band in the region of $740-850 \mathrm{~cm}^{-1}$, originating from $-\mathrm{CH}_{3}$ rocking and $\mathrm{Si}-\mathrm{C}$ stretching is also clearly visible. This absorption peak became broader and the peak height decreased gradually with increasing the mol\% of $\mathrm{CF}_{3}$ groups. Additionally, the main absorption peak of Si-C bond shifted to a slightly higher frequency, as did the absorption peak of $\mathrm{C}-\mathrm{H}$ bonds in $-\mathrm{CH}_{3}$, which further supports the presence of the $\mathrm{CF}_{3}$ units. Furthermore, all the absorption intensities of the characteristic peaks of $\mathrm{CF}_{3}$ increased with increasing the feed ratio of $F_{3}$, which suggested that the content of $\mathrm{CF}_{3}$ groups in $\mathbf{P}_{\mathbf{x}}$ increased along with increasing the $\mathrm{F}_{3}$ feed ratio. The presence of vinyl groups is confirmed by a broad and weak absorption band from $1600 \mathrm{~cm}^{-1}$ to $1720 \mathrm{~cm}^{-1}$ present in each spectrum. ${ }^{32}$ As mentioned above, the end-functionalization of $\mathbf{P}_{\mathbf{x}}$ with vinyl groups is important, since these groups are used to crosslink $\mathbf{P}_{\mathbf{x}}$ in thin films.

The thermal stability of $\mathbf{P}_{\mathbf{x}}$ was investigated by TGA in argon atmosphere and shows that $\mathbf{P}_{\mathbf{x}}$ are stable up to $380{ }^{\circ} \mathrm{C}$ and have a maximum weight loss around $450{ }^{\circ} \mathrm{C}$ (Fig. 2).

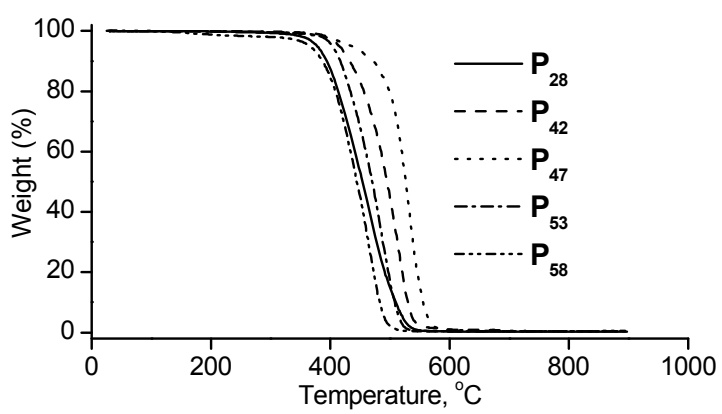

Fig. 2 TGA curves of $P_{x}$ conducted at a heating rate of $20^{\circ} \mathrm{C} \mathrm{min}^{-1}$ under nitrogen.

Differential scanning calorimetry (DSC) measurements show an increase in $T_{g}$ with increasing the mol\% of $\mathrm{CF}_{3}$ groups from $-106{ }^{\circ} \mathrm{C}$ for $\mathbf{P}_{\mathbf{2 8}}$ to $-88.6{ }^{\circ} \mathrm{C}$ for $\mathbf{P}_{\mathbf{5 8}}$ (ESI Fig. S12-S16, Table 4). It is known that the introduction of polar moieties in a polymer shifts the $T_{g}$ of the modified polymer to higher values. Luckily, the $T_{g} \mathbf{S}$ of $\mathbf{P}_{\mathbf{x}}$ are well below room temperature and thus the materials obtained by crosslinking $\mathbf{P}_{\mathbf{x}}$ are still elastic. The presence of only one glass transition temperature indicates that the distribution of $\mathrm{CF}_{3}$ groups in $\mathbf{P}_{\mathbf{x}}$ is random. No melting temperatures were observed in the DSC. PDMS is known to easily crystallize at about $-80{ }^{\circ} \mathrm{C} .{ }^{33}$ However, by modifying the silicone with $\mathrm{CF}_{3}$ group, the crystallization process in $\mathbf{P}_{\mathbf{x}}$ is hindered. Even for $\mathbf{P}_{\mathbf{2}}$, which has the lowest content of $\mathrm{CF}_{3}$ groups, no melting temperature was observed. ${ }^{34}$

Table 4 Transition temperatures observed for $\mathbf{P}_{\mathbf{x}}$ as well as the $\Delta \mathrm{C}_{\mathrm{p}}$ of the transitions.

\begin{tabular}{ccccc}
\hline Copolymer & \multicolumn{2}{c}{$T_{g}\left[{ }^{\circ} \mathrm{C}\right]$} & \multicolumn{2}{c}{$\Delta \mathrm{C}_{\mathrm{p}}\left[\mathrm{J} \mathrm{g}^{-1}{ }^{\circ} \mathrm{C}^{-1}\right]$} \\
& $2^{\text {nd }}$ heating & cooling & $2^{\text {nd }}$ heating & cooling \\
\hline $\mathbf{P}_{\mathbf{2 8}}$ & -106.0 & -115.4 & 0.39 & 0.47 \\
$\mathbf{P}_{\mathbf{4 2}}$ & -97.7 & -105.8 & 0.27 & 0.28 \\
$\mathbf{P}_{\mathbf{4 7}}$ & -97.1 & -103.6 & 0.30 & 0.25 \\
$\mathbf{P}_{\mathbf{5 3}}$ & -92.2 & -98.0 & 0.33 & 0.24 \\
$\mathbf{P}_{\mathbf{5 8}}$ & -88.6 & -95.8 & 0.31 & 0.25 \\
\hline
\end{tabular}

\section{Synthesis and characterization of $P_{x}(y)$}

All materials of series $\mathbf{P}_{\mathbf{x}}$ are highly viscous liquids and have to be cross-linked to furnish elastomers. As mentioned before, we designed $\mathbf{P}_{\mathbf{x}}$ with vinyl end-groups which should allow cross-linking. Next, the vinyl end-functionalized $\mathbf{P}_{\mathbf{x}}$ were cross-linked via hydrosilylation using a tetrafunctional cross-linker tetrakis(dimethylsiloxy)silane and Karstedt's platinum catalyst. The molar ratio of the vinyl groups of $\mathbf{P}_{\mathbf{x}}$ to the hydrosilyl groups of the $\mathrm{CL}$ was kept constant (1.1) for all synthesized materials except for $\mathbf{P}_{47}$ for which the ratio was increased. The reason for this was the fast cross-linking observed when $\mathbf{P}_{\mathbf{4}}$ was cross-linked, which did not allow sufficient time for thin film preparation. A reduction of the catalyst did not reduce the reaction rate significantly. Therefore the amount of cross-linker used had to be decreased. To optimize the mechanical properties of the resulting materials, hexamethyldisilazane treated silica particles were used as reinforcing filler. Thin films were prepared by the doctor blade technique on a Teflon substrate. The synthesized materials were named as $\mathbf{P}_{\mathbf{x}}(\mathbf{y})$, where $\mathbf{P}_{\mathbf{x}}$ represents the copolymer and $\mathbf{y}$ represents the $w t \%$ of surface treated silica particles used.

The mechanical properties of $\mathbf{P}_{\mathbf{x}}(\mathbf{y})$ were investigated in tensile tests (see ESI Fig. S17-S28). Fig. 3 shows the stress-strain curves of materials $\mathbf{P}_{\mathbf{x}}(\mathbf{y})$, while Table 5 gives an overview of the Young's moduli at different strains $\left(Y_{X \%}\right)$ and the strain at break. It is known that the mechanical properties of a material are strongly affected by the molecular weight of the polymer used. Unfortunately, because the molar masses of $\mathbf{P}_{\mathbf{x}}$ were rather different, a direct comparison of the mechanical properties as function of $\mathrm{CF}_{3}$ content was not possible. However, irrespective of the content of trifloropropyl units in the materials and the $M_{w}$ of the polymer used, an increase in the tensile strength and a decrease in the elongation at break with increasing amount of silica were observed. Materials $\mathbf{P}_{\mathbf{x}}(\mathbf{y})$, for which the concentration of cross-linker to vinyl groups was kept constant, showed an increase in the strain at break with increasing the molar mass of polymer $\mathbf{P}_{\mathbf{x}}$ used. The lowest strain at break of $180 \%$ was observed for material $\mathbf{P}_{\mathbf{4 7}}(\mathbf{1 0})$ which was synthesized starting from $\mathbf{P}_{\mathbf{4}}$ and had the lowest $M_{w}$, while the maximum strain at break of $850 \%$ was observed for $\mathbf{P}_{\mathbf{4 2}}(\mathbf{0})$ which was prepared starting from $\mathbf{P}_{\mathbf{4 2}}$ and had the highest $M_{w}$. All other materials showed an elongation at break value between 240 to $550 \%$. It should be mentioned here that materials $\mathbf{P}_{\mathbf{4 2}}(\mathbf{y})$ showed visible viscous flow when strained, were sticky and difficult to handle in thin films. For materials $\mathbf{P}_{58}(\mathbf{y})$ which have the highest $\mathrm{CF}_{3}$ content, the strain at break was slightly improved when $5 \mathrm{wt} \%$ silica was used and the tensile strength was increased from $0.05 \mathrm{MPa}$ for $\mathbf{P}_{58}(\mathbf{0})$ to $0.22 \mathrm{MPa}$ for $\mathbf{P}_{\mathbf{5 8}}(\mathbf{5})$. Sample $\mathbf{P}_{\mathbf{5 3}}(\mathbf{5})$ showed an increased tensile strength, while the strain at break was only slightly affected as compared to $\mathbf{P}_{53}(\mathbf{0})$. Furthermore, an increase in the stickiness with $\mathrm{CF}_{3}$ content was observed. All obtained materials $\mathbf{P}_{\mathbf{x}}(\mathbf{y})$ are 
rather soft and have Young's moduli (at 10\% strain) that range moduli with the addition of silica filler was observed (see Table 5). between $19 \mathrm{kPa}$ to $405 \mathrm{kPa}$. Additionally an increase in the Young's
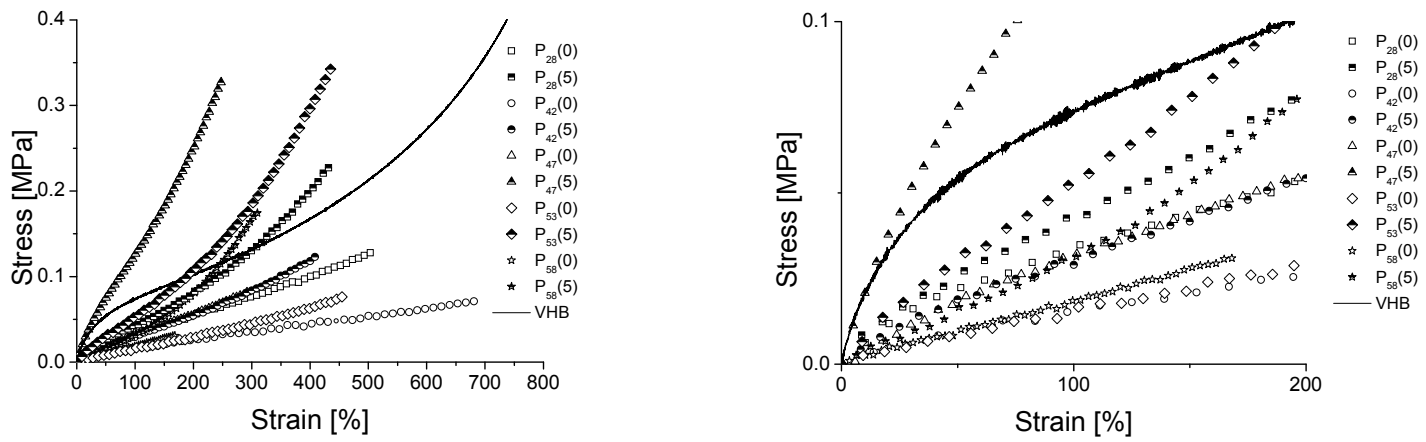

Fig. 3 Stress-strain curves for $\mathbf{P}_{\mathbf{x}}(\mathbf{y})$ (left) with the stress-strain curves up to $200 \%$ strain enlarged (right). The strain at break represents the lowest value obtained from different measurements. Tensile test specimens with a gauge width of $2 \mathrm{~mm}$ and a gauge length of $18 \mathrm{~mm}$ were tested at a crosshead speed of $200 \mathrm{~mm} \mathrm{~min}^{-1}$.

Table 5 Overview of the Young's moduli at different strains and the strain at break for $\mathbf{P}_{\mathbf{x}}(\mathbf{y})$.

\begin{tabular}{|c|c|c|c|c|c|}
\hline \multirow[b]{2}{*}{ Sample } & \multicolumn{4}{|c|}{ Young Modulus [kPa] ${ }^{\mathrm{a}}$} & \multirow{2}{*}{$\begin{array}{c}\text { Strain break } \\
{[\%]}\end{array}$} \\
\hline & $\begin{array}{c}10 \% \\
(0-20)\end{array}$ & $\begin{array}{c}50 \% \\
(40-60)\end{array}$ & $\begin{array}{c}100 \% \\
(90-110)\end{array}$ & $\begin{array}{c}200 \% \\
(190-210)\end{array}$ & \\
\hline$P_{28}(0)$ & 58 & 26 & 22 & 21 & 513 \\
\hline$P_{28}(5)$ & 65 & 35 & 34 & 44 & 488 \\
\hline$P_{28}(10)$ & 97 & 42 & 46 & 61 & 552 \\
\hline $\mathbf{P}_{42}(0)$ & 24 & 13 & 11 & 10 & 850 \\
\hline$P_{42}(5)$ & 45 & 26 & 22 & 27 & 540 \\
\hline$P_{47}(0)$ & 50 & 30 & 24 & 25 & 360 \\
\hline$P_{47}(5)$ & 184 & 107 & 104 & 150 & 240 \\
\hline$P_{47}(10)$ & 405 & 298 & 352 & - & 180 \\
\hline$P_{53}(0)$ & 19 & 15 & 15 & 13 & 498 \\
\hline$P_{53}(5)$ & 72 & 46 & - & - & 459 \\
\hline$P_{58}(0)$ & 20 & 17 & 18 & - & 263 \\
\hline$P_{58}(5)$ & 34 & 28 & 34 & 64 & 349 \\
\hline VHB & b146 & 50 & 30 & 27 & \\
\hline
\end{tabular}

a) Young's moduli at different strain levels; ${ }^{b}$ the slope between 5 and $15 \%$ strain was taken, ${ }^{c}$ the average of the strain at break from different measurements is given.

The dielectric properties of materials $\mathbf{P}_{\mathbf{x}}(\mathbf{y})$ were also measured in a frequency range from $10^{-1} \mathrm{~Hz}$ to $10^{6} \mathrm{~Hz}$ and room temperature (Fig. 4). Table 6 summarizes $\varepsilon^{\prime}$, dielectric loss $\left(\varepsilon^{\prime \prime}\right)$ and conductivity $(\sigma)$ at 10 $\mathrm{kHz}$ for materials $\mathbf{P}_{\mathbf{x}}(\mathbf{0}), \mathbf{P}_{\mathbf{x}}(\mathbf{5})$ and $\mathrm{VHB}$ foil. $\boldsymbol{\varepsilon}^{\prime}$ raised with increasing amounts of $\mathrm{CF}_{3}$ groups in the materials. The $\varepsilon^{\prime}$ values are almost constant at frequencies above $10^{2} \mathrm{~Hz}$. The increase in $\varepsilon^{\prime}$ at low frequencies is most likely caused by electrode polarization. ${ }^{35}$ The $\varepsilon^{\prime}$ at $10 \mathrm{kHz}$ for $\mathbf{P}_{\mathbf{x}}(\mathbf{y})$ as well as for $\mathbf{F}_{\mathbf{3}}$ monomer $\left(\varepsilon^{\prime}=8.8\right)$ are given as function of $\mathrm{CF}_{3}$ content in Fig. 5. A linear increase in $\varepsilon^{\prime}$ with increasing amounts of $\mathrm{CF}_{3}$ groups from $\varepsilon^{\prime}=5.1$ for $\mathbf{P}_{28}(0)$ to $\varepsilon^{\prime}=6.4$ for $\mathbf{P}_{58}(\mathbf{0})$ was observed. Since the permittivity is increasing with the concentration of the polar groups in the material, it is expected that a material $\mathbf{P}_{\mathbf{1 0 0}}(\mathbf{0})$ would have a maximum $\varepsilon^{\prime}=8.8$. Materials $\mathbf{P}_{\mathbf{x}}(\mathbf{5})$ and $\mathbf{P}_{\mathbf{x}}(\mathbf{1 0})$ which contain 5 and $10 \mathrm{wt} \%$ silica particles show slightly lower values for the permittivity as compared to $\mathbf{P}_{\mathbf{x}}(\mathbf{0})$. This decrease is not unexpected given the fact that silica has $\varepsilon^{\prime}=3.9$. The conductivity at low frequencies (the static conductivity) is lower than $10^{-10} \mathrm{~S} / \mathrm{cm}$, typical for insulating materials. ${ }^{36}$ The silica modified materials $\mathbf{P}_{\mathbf{x}}(\mathbf{5})$ and $\mathbf{P}_{\mathbf{x}}(\mathbf{1 0})$, tend to have a lower conductivity than $\mathbf{P}_{\mathbf{x}}(\mathbf{0})$ (Fig. 4).

In all materials $\mathbf{P}_{\mathbf{x}}(\mathbf{y})$, a minimum in the dielectric losses of less than $10^{-2}$ was observed between $10^{4}$ and $10^{5} \mathrm{~Hz}$ which recommends these materials for high frequency applications.

Table 6 Dielectric properties, actuation strain $(s)$ at $7.8 \mathrm{~V} / \mu \mathrm{m}$, maximum actuation strain $\left(s_{\max }\right)$, and dielectric breakdown in actuator $\left(E_{b}\right)$ for $\mathbf{P}_{\mathrm{x}}(\mathbf{y})$

\begin{tabular}{|c|c|c|c|c|c|c|c|c|c|c|}
\hline Sample & ${ }^{\mathrm{a}} \varepsilon^{\prime}$ & ${ }^{\mathrm{a}} \varepsilon^{\prime \prime}$ & $\begin{array}{c}{ }^{\mathrm{a}} \sigma \\
{\left[\mathrm{S} \mathrm{cm}^{-1}\right]}\end{array}$ & $\operatorname{Tan} \delta$ & $\begin{array}{c}Y_{10 \%} \\
{[\mathrm{kPa}]}\end{array}$ & $\begin{array}{c}\varepsilon^{\prime} / Y \\
{\left[\mathrm{MPa}^{-1}\right]}\end{array}$ & $\begin{array}{l}s[\%] \\
\text { at } 7.8 \\
V / \mu m\end{array}$ & $\begin{array}{l}\mathrm{s}_{\max } \\
{[\%]}\end{array}$ & $\begin{array}{c}E_{b} \\
{[\mathrm{~V} / \mu \mathrm{m}]}\end{array}$ & $\begin{array}{c}{ }^{d} d \\
{[\mu \mathrm{m}]}\end{array}$ \\
\hline
\end{tabular}




\begin{tabular}{|c|c|c|c|c|c|c|c|c|c|c|}
\hline$P_{28}(0)$ & 5.1 & 0.018 & $8.92 \times 10^{-11}$ & 0.0035 & 58 & 88 & 1.1 & 4.8 & 19.1 & 89 \\
\hline$P_{28}(5)$ & 4.5 & 0.005 & $2.95 \times 10^{-11}$ & 0.0012 & 65 & 69 & - & - & - & - \\
\hline$P_{42}(0)$ & 5.6 & 0.004 & $1.89 \times 10^{-11}$ & 0.0007 & 24 & 233 & - & 1.8 & 6.5 & 175 \\
\hline$P_{42}(5)$ & 5.3 & 0.004 & $2.40 \times 10^{-11}$ & 0.0008 & 45 & 118 & 0.8 & 5.1 & 17.1 & 317 \\
\hline$P_{47}(0)$ & 5.7 & 0.007 & $3.37 \times 10^{-11}$ & 0.0012 & 50 & 114 & 0.9 & 3.7 & ${ }^{b} 16.0$ & 317 \\
\hline$P_{47}(5)$ & 5.7 & 0.004 & $2.03 \times 10^{-11}$ & 0.0007 & 184 & 31 & - & - & - & - \\
\hline$P_{53}(0)$ & 6.2 & 0.005 & $2.35 \times 10^{-11}$ & 0.0008 & 19 & 326 & 5.4 & 5.4 & 7.8 & 320 \\
\hline$P_{53}(5)$ & 6.0 & 0.005 & $2.43 \times 10^{-11}$ & 0.0009 & 72 & 83 & 1.1 & 3.6 & 13.3 & 223 \\
\hline$P_{58}(0)$ & 6.4 & 0.007 & $3.37 \times 10^{-11}$ & 0.00011 & 20 & 320 & - & - & - & - \\
\hline$P_{58}(5)$ & 6.1 & 0.005 & $2.66 \times 10^{-11}$ & 0.0009 & 34 & 179 & 3.1 & 5.0 & 10.2 & 157 \\
\hline VHB & 4.4 & - & $9.02 \times 10^{-10}$ & 0.044 & ${ }^{c} 32$ & 138 & - & - & $>100$ & - \\
\hline
\end{tabular}

${ }^{\text {a) }}$ The permittivity, dielectric losses and conductivity values were taken at $10 \mathrm{kHz} ;{ }^{\mathrm{b}}$ maximum field achieved, no breakdown; ${ }^{\mathrm{c}} Y$ at $300 \% \mathrm{strain}$; dactuator thickness.

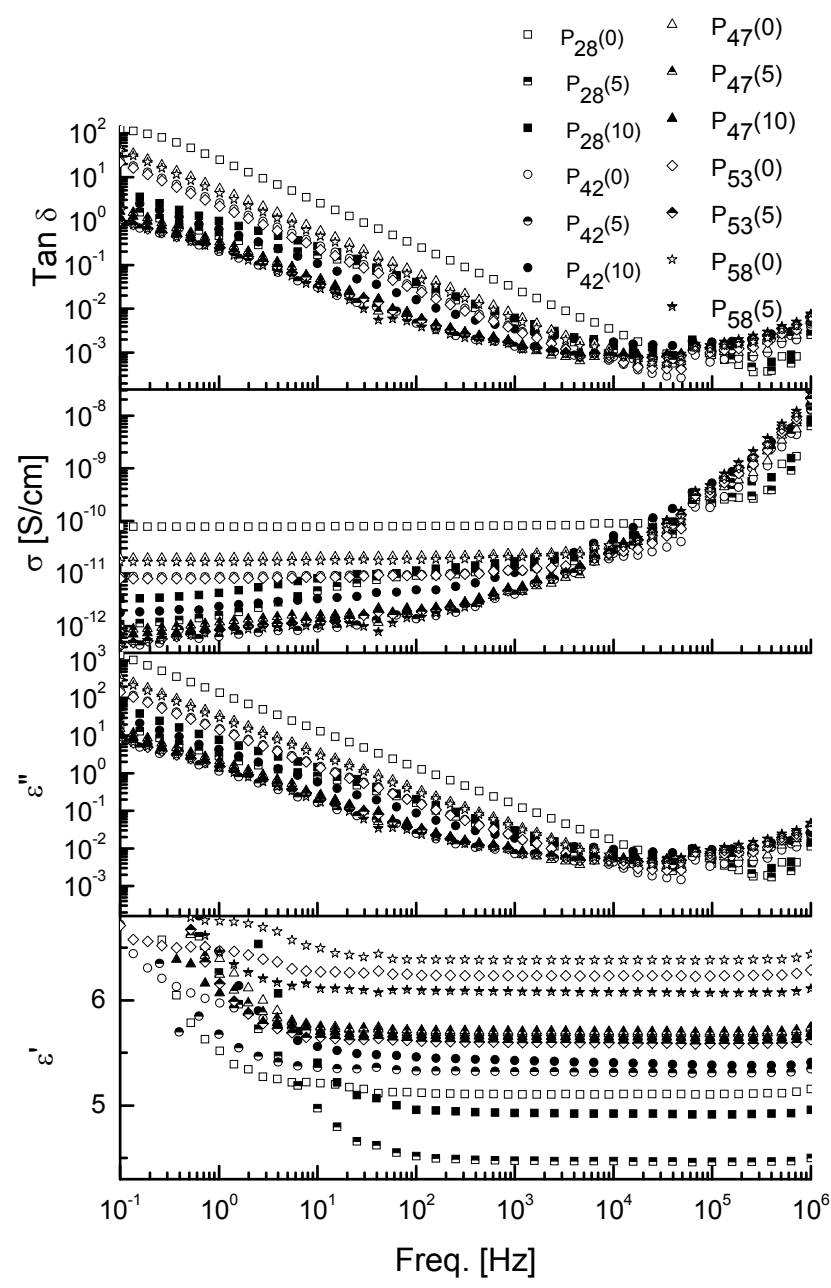

Fig. 4 Dielectric properties as function of frequency of $\mathbf{P}_{\mathbf{x}}(\mathbf{y})$.

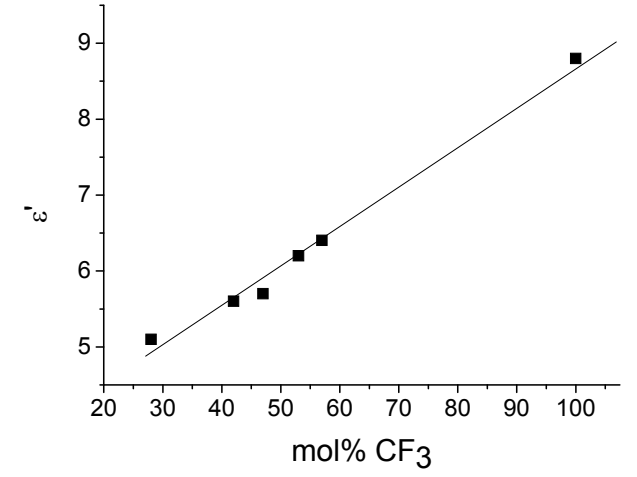

Fig. 5 Dielectric permittivity at $10 \mathrm{kHz}$ of $\mathbf{P}_{\mathbf{x}}(\mathbf{y})$ as function of mol\% of $\mathrm{CF}_{3}$ groups. The permittivity at $100 \%$ is the permittivity of monomer $\mathrm{F}_{3}$.

Some materials were further investigated in electromechanical tests using circular actuators. The voltage was increased in steps of $100 \mathrm{~V}$ every $2 \mathrm{~s}$ until breakdown through the material occurred. Fig. 6 shows the lateral actuation strain as a function of the electric field, while Table 6 summarizes the maximum actuation strain achieved at breakdown, and the actuation strains at $7.8 \mathrm{~V} / \mu \mathrm{m}$ for $\mathbf{P}_{\mathbf{x}}(\mathbf{y})$. All tested materials showed actuation at rather low electric fields of less than $20 \mathrm{~V} / \mu \mathrm{m}$. The best performance in terms of actuation strain at a low electric field was observed for material $\mathbf{P}_{53}(\mathbf{0})$ which has the highest ratio of $\varepsilon^{\prime} / Y$ and showed an actuation strain of $5.4 \%$ at $7.8 \mathrm{~V} / \mu \mathrm{m}$, while material $\mathbf{P}_{53}(\mathbf{5})$ showed significantly lower actuation strain of $1.1 \%$. The reason behind this is its slightly lower permittivity value and higher Young's moduli. Material $\mathbf{P}_{\mathbf{5 8}} \mathbf{( 5 )}$ showed slightly lower actuation strain as compared to $\mathbf{P}_{\mathbf{5 3}}(\mathbf{0})$ most likely due to its higher elastic modulus. The lowest actuation strain was observed for material $\mathbf{P}_{\mathbf{2 8}}(\mathbf{0})$ which had the lowest content of $\mathrm{CF}_{3}$.

$A$ raise in the actuation strain at a certain electric field with increasing the content of $\mathrm{CF}_{3}$ groups was observed for materials $\mathbf{P}_{\mathbf{x}}(\mathbf{5})$. For example, materials $\mathbf{P}_{\mathbf{4 2}}(\mathbf{5})$ and $\mathbf{P}_{\mathbf{5 8}}(\mathbf{5})$ showed about the same actuation strain of $5 \%$ but the electric field used decreased from $17.1 \mathrm{~V} / \mu \mathrm{m}$ for $\mathbf{P}_{\mathbf{4 2}}(\mathbf{5})$ to $10.2 \mathrm{~V} / \mu \mathrm{m}$ for $\mathbf{P}_{\mathbf{5 8}}(\mathbf{5})$, respectively. Material $\mathbf{P}_{\mathbf{2 8}}(\mathbf{0})$ shows the highest breakdown field of $19.1 \mathrm{~V} / \mu \mathrm{m}$. The film prepared from material $\mathbf{P}_{\mathbf{4 7}}(\mathbf{0})$ was rather thick $(317 \mu \mathrm{m})$ and therefore, the maximum given actuation is not the actuation at breakdown. Furthermore, a decrease in the actuator breakdown 
field with increasing the content of polar component from 19.1 $\mathrm{V} / \mu \mathrm{m}$ for $\mathbf{P}_{28}(\mathbf{0})$ to $7.8 \mathrm{~V} / \mu \mathrm{m}$ for $\mathbf{P}_{53}(\mathbf{0})$ was observed.

As mentioned above, materials $\mathbf{P}_{\mathbf{4 2}}(\mathbf{y})$ after straining, do not immediately recover their initial shape. Room temperature rheological measurements show that the storage moduli $\mathrm{G}^{\prime}$ for the tested material are rather low, typical for elastomeric materials (see DMA in ESI Fig. S29). The viscoelastic losses of different materials negatively affect the cyclic actuation strain at a certain frequency (see ESI, Fig. S30-S33).

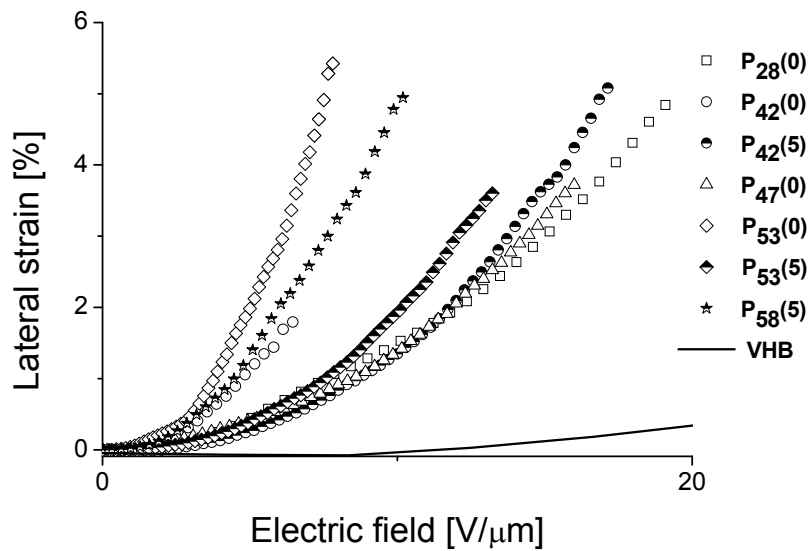

Fig. 6 Lateral actuation strain of circular actuators of materials $\mathbf{P}_{\mathbf{x}}(\mathbf{y})$, as a function of applied electric field. The voltage was increased by $100 \mathrm{~V}$ every $2 \mathrm{~s}(0.5 \mathrm{~Hz})$. The actuator constructed from $\mathbf{P}_{47}(\mathbf{0})$ did not suffer a breakdown at $16 \mathrm{~V} / \mu \mathrm{m}$, however, we could not increase the voltage further due to the limitation of the voltage source used.

For example, while the cyclic actuation of $\mathbf{P}_{\mathbf{4 7}}(\mathbf{0})$ at $17.6 \mathrm{~V} / \mu \mathrm{m}$ showed only a small hysteresis in the actuation strain in time (Fig. 7, Fig S34), materials $\mathrm{P}_{42}(\mathrm{y})$ showed quite some hysteresis and require a few cycles to achieve a stable actuation (see ESI, Fig. S31, S31). Cyclic actuation tests for materials $\mathbf{P}_{\mathbf{5 3}}(\mathbf{y})$ and $\mathbf{P}_{\mathbf{5 8}}(\mathbf{5})$ also show a rather small hysteresis (see ESI, Fig. S35-S37). Some actuators, especially those constructed from materials with high $\mathrm{CF}_{3}$ content showed a repairing effect which was observed during the cyclic actuation tests (see ESI, Fig. S36). Although this effect is of potential advantage since the lifetime and the reliability of the actuator is increased, this process was rather slow and can occasionally take minutes.

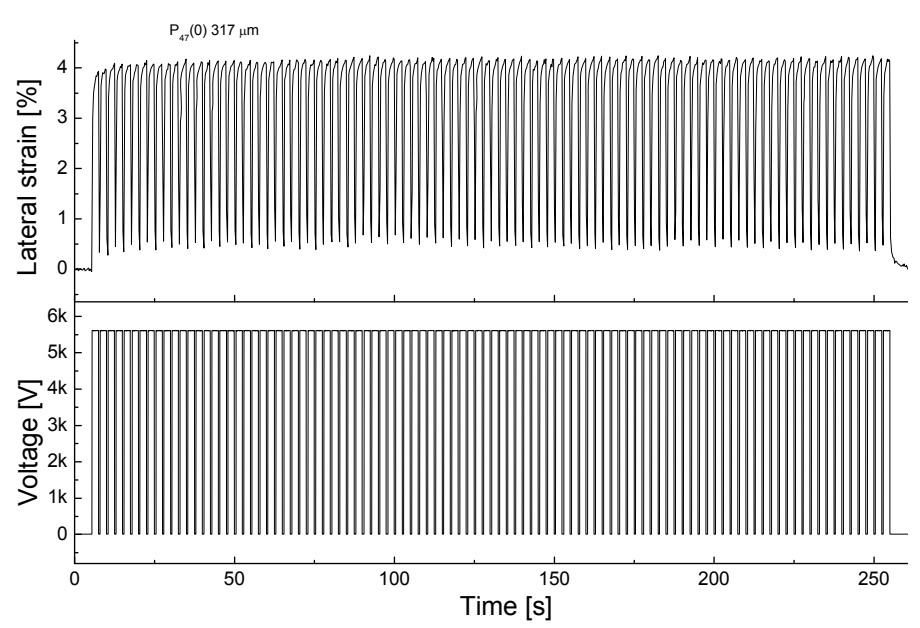

Fig. 7 Long-term stability of $\mathbf{P}_{\mathbf{4} 7}(\mathbf{0})$ actuator at $17.6 \mathrm{~V} / \mu \mathrm{m}$ at $28.6 \%$ prestrain (100 cycles at $0.4 \mathrm{~Hz}$ ).

Finally, the performance of our material will be compared to other reported materials ${ }^{26}$ and VHB. An elastomer containing $45 \mathrm{wt} \% \mathrm{CF}_{3}$ modified silicone which represents about $28 \mathrm{~mol} \%$ of $\mathrm{CF}_{3}$ units in the final material has a similar chemical composition as $\mathbf{P}_{\mathbf{2 8}}(\mathbf{0})$. The reported material has a $\varepsilon^{\prime}=5.4$, which is in agreement with the values measured by us $\left(\varepsilon^{\prime}=5.1\right)$. While the reported material has a strain at break of $280 \%, \mathbf{P}_{28}(0)$ shows a much higher strain at break of $513 \%$. Unfortunately, the actuation strain of the two materials cannot be easily compared, since different setups were used. The VHB foil is widely used by the DEA community and it was therefore selected here as reference material. The mechanical and dielectric characteristics of VHB were included in Tables 5 and $6 .{ }^{37}$ The VHB membrane was prestrained biaxially by $300 \%$ before circular carbon black electrodes with a diameter of $8 \mathrm{~mm}$ were applied. As can be seen in Fig. 6, by far the lowest actuation strain at a certain electric field is observed for VHB which at $20 \mathrm{~V} / \mu \mathrm{m}$ showed only a $0.35 \%$ lateral actuation strain. However, in terms of maximum achievable actuation strain and dielectric breakdown, the VHB foil is still unbeatable. However, for implantable devices for which the maximum allowed voltage is $24 \mathrm{~V}$, the materials presented herein are superior. Future work will focus on the upscaling of the synthesis and the optimization of the mechanical properties of materials $\mathbf{P}_{\mathbf{5 3}}(\mathbf{y})$ and $\mathbf{P}_{\mathbf{5 8}}(\mathbf{y})$ as well as on investigating how the dielectric breakdown of these materials can be increased.

\section{Conclusions}

Polysiloxanes $\mathbf{P}_{\mathbf{x}}$ containing different mol\% of trifluoropropyl groups and vinyl end-groups were successfully obtained under anionic conditions starting from $F_{3}, D_{4}$, and 1,3-divinyl-1,1,3,3tetramethyldisiloxane end-capping reagent. By cross-linking $\mathbf{P}_{\mathbf{x}}$ in thin films using a hydrosilylation reaction, elastomers with good mechanical and dielectric properties were obtained. All the materials show a strain at break higher than $180 \%$. The highest strain at break value was $850 \%$ and was obtained for a material for which a high molecular weight $\mathbf{P}_{\mathbf{4 2}} \mathbf{( 0 )}$ was used. The dielectric permittivity raised with increasing amounts of polar trifluoropropyl groups and a maximum value of $\varepsilon^{\prime}=6.4$ was measured for $\mathbf{P}_{58}(\mathbf{0})$. An actuation strain of $5.4 \%$ at $7.8 \mathrm{~V} / \mu \mathrm{m}$ where electrical breakdown 
occurred was measured for a film prepared starting from $\mathbf{P}_{53}(\mathbf{0})$. The increased permittivity and the low actuation voltage recommend these materials as dielectrics in sensors and actuators.

\section{Acknowledgements}

We gratefully acknowledge Sciex (project no. 12.192, the Swiss National Science Foundation (IZERZO - 142215/1 and No. 150638), and the Swiss Federal Laboratories for Materials Science and Technology for financial support. We also like to acknowledge B. Fischer (Empa, Switzerland) for the TGA and DSC measurements, D. Rentsch (Empa, Switzerland) for helping with the NMR measurements, and G. Kovacs (Empa, Switzerland) for providing us with the infrastructure for the actuator measurements. DMO thanks C. Racles from Petru Poni Institute, lasi, Romania for her kind support of the Sciex fellow and Prof. F. Nüesch from Empa for his generous support.

\section{Notes and references}

1. P. Brochu, Q. Pei, Macromol. Rapid Commun. 2010, 31, 1036.

2. J. Biggs, K. Danielmeier, J. Hitzbleck, J. Krause, T. Kridl, S. Nowak, E. Orselli, X. Quan, D. Schapeler, W. Sutherland, J. Wagner, Angew. Chem. Int. Ed. 2013, 52, 9409-9421.

3. R. Perline, R. Kornbluh, Q. Pei, J. Joseph, Science, 2000, 287, 836-839.

4. F. Carpi, D. De Rossi, R. Kornbluh, R. Pelrine, P. SommerLarsen, in Dielectric Elastomers as Electromechanical Transducers: Fundamentals, Materials, Devices, Models and Applications of an Emerging Electroactive Polymer Technology, Elsevier, Oxford, 2011.

5. M. lacob, A. Bele, X. Patras, S. Pasca, M. Butnaru, M. Alexandru, D. Ovezea, M. Cazacu, Mater. Sci. Engineering, 2014, 43, 392-402.

6. S. Akbari, H. R. Shea, Sens. Actuat. A 2012, 186, 236-241.

7. C. Racles, M. Cazacu, B. Fischer, D. M. Opris, Smart. Mater. Struct. 2013, 22, 104004.

8. B. Müller, H. Deyhle, S. Mushkolaj, M. Wieland, Swiss Med. Wkly. 2009, 139, 591.

9. S. Rudykh, A. Lewinstein, G. Uner, G. deBotton, Appl. Phys. Lett. 2013, 102, 151905.

10. G. Gallone, F. Carpi, F. Galantini, D. De Rossi, G. Levita, Adv. Sci. Tech. 2008, 61, 46-52.

11. H. Stoyanov, M. Kollosche, S. Risse, D. N. McCarthy, G. Kofod, Soft Matter, 2011, 7, 194.

12. C. Huang, Q. M. Zhang, G. deBotton, K. Bhattacharya, Appl. Phys. Lett. 2004, 84, 4391.

13. M. Molberg, D. Crespy, P. Rupper, F. Nüesch, J.-A. E. Månson, C. Löwe, D. M. Opris, Adv. Funct. Mater. 2010, 20, 3280-3291.

14. J. E. Q. Quinsaat, M. Alexandru, F. A. Nüesch, H. Hofmann, A. Borgschulte, D. M. Opris, , J. Mater. Chem. A, 2015, 3, 14675-14685.

15. H. Stoyanov, D. McCarthy, M. Kollosche, G. Kofod, Appl. Phys. Lett. 94, 202901/1.
16. T. P. Selvin, A. A. Abdullateef, M. A. Al-Harthi, M. A. Atieh, S. K. De, M. Rahaman, T. K. Chaki, D. Khastgir, S. Bandyopadhyay, J. Mater. Sci. 2012, 47, 3344-3349.

17. F. Carpi, G. Gallone, F. Galantini, D. De Rossi, Adv. Funct. Mater. 2008, 18, 235

18 Feast, W. J.; Gimeno, M.; Khosravi, E. Polymer, 2003, 44, 6111-6121.

19. F. B. Madsen , I. Dimitrov , A. E. Daugaard, S. Hvilsted, A. L. Skov, Polym. Chem., 2013, 4, 1700-1707.

20. B. Kussmaul, S. Risse, G. Kofod, R. Waché, M. Wegener, D. N. McCarthy, H. Krüger, R. Gerhard, Adv. Funct. Mater. 2011, 21, 4589-4594.

21. F. B. Madsen, L. Yu, A. E. Daugaard, S. Hvilsted, A. L. Skov, RSC Adv., 2015, 5, 10254-10259

22. S. J. Dünki, M. Tress, F. Kremer, S. Y. Ko, F. A. Nüesch, C.-D. Varganici, C. Racles, D. M. Opris, RSC Adv., 2015, 5, 50054-50062.

23. C. Racles, M. Alexandru, A. Bele, V. E. Musteata, M. Cazacu, D. M. Opris, RSC Adv., 2014, 4, 37620-37628.

24. S. J. Dünki , Y. S. Ko , F. A. Nüesch, D. M. Opris, Adv. Funct. Mater. 2015, 25, 2467-2475.

25. R. Pelrine, R. Kornbluh, J. Joseph, R. Heydt, Q. Pei, S. Chiba, Mater. Sci. Eng. C, 2000, 11, 89-100

26. H. Böse, D. Uhl, R. Rabindranath, Proc. of SPIE, 2012, 8340, 83402E-1.

27. M. Fujino, T. Hisaki, M. Fujiki, N. Matsumoto, Macromolecules, 1992, 25, 1079-1083.

28. J. Chojnowski, M. Cypryk, W. Fortuniak, M. Scibiorek and K. Rozga-Wijas, Macromolecules, 2003, 36, 38903897.

29. M. Cypryk, B. Delczyk, A. Juhari, K. Koynov, J. Pol. Sci. A: Pol. Chem. 2009, 47, 1204-1216.

30. L. M. Yi, X. L. Zhan, F. Q. Chen, F. Du, L. B. Huang, J. Polym. Sci., Part A: Polym. Chem., 2005, 43, 44314438.

31. A. S. Palsule, Y. Poojari, Polymer, 2010, 51, 6161-6167.

32. H. Li, L. Zhang, L. Cheng, Y. Wang, Z. Yu, M. Huang, H. Tu, H. Xia, J. Mater. Sci., 2008, 43, 2806-2811.

33. M. Alexandru, M. Cristea, M. Cazacu, A. Ioanid, B.C. Simionescu, Polym. Composites, 2009, 30, 751.

34. J. Chojnowski, M. Cypryk, W. Fortuniak, M. Scibiorek, K. Rozga-Wijas, Macromolecules, 2003, 36, 3890-3897.

35. A. K. Jonscher, J. Phys. D: Appl. Phys., 1999, 32, R57R70.

36. J. W. Williams. J. Phys. Chem., 1932, 36, 437-443.

37. For DMA characterization and cyclic actuation tests of VHB foil, the reader is referred to: D. M. Opris, M. Molberg, C. Walder, Y. S. Ko, B. Fischer, F. A. Nüesch, Adv. Funct. Mater. 2011, 21, 3531-3539. 